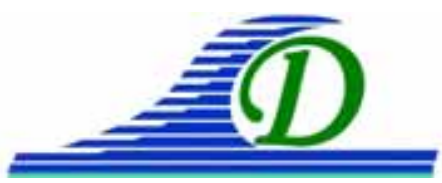

XIII ${ }^{\text {èmes }}$ Journées Nationales Génie Côtier - Génie Civil

Dunkerque, 2-4 juillet 2014

DOI:10.5150/jngcgc.2014.021 @ Editions Paralia CFL

disponible en ligne - http://www.paralia.fr - available online

\title{
Modélisation hydrodynamique sur le littoral de la région des Pays de la Loire
}

\section{Laura TROUDET $^{1}$, Stéphanie DESMARE ${ }^{1}$}

\author{
1. SHOM, Département Hydrodynamique Côtière, \\ 13 rue du Chatellier, CS 92803, 29228 Brest, France. \\ laura.troudet@shom.fr
}

\section{Résumé :}

Un modèle hydrodynamique tridimensionnel a été développé au SHOM, dans le cadre d'une convention de partenariat entre l'Europe, l'Etat, la Région des Pays de la Loire et le SHOM, pour favoriser l'exploitation d'Energies Marines Renouvelables (EMR). Le modèle reproduit l'évolution des courants de marée sur la colonne d'eau sur l'ensemble du littoral de la région des Pays de la Loire, incluant l'estuaire de la Loire. Le calcul repose sur un système de modélisation aux éléments finis TELEMAC-3D (EDF, 2013) qui s'appuie sur un maillage triangulaire mixte et des plans sur la verticale en transformation sigma. Un forçage de marée et fluvial est appliqué aux conditions aux limites.

Le calage du modèle est réalisé à la fois en niveau d'eau et vitesse sur une période réelle. Les données de références utilisées pour le calage sont les observations du niveau d'eau issues des marégraphes du SHOM mais aussi des mesures de partenaires REFMAR, des prédictions de marée du SHOM, et des mesures de courants. Les simulations donnent de bons résultats quantifiés par des méthodes statistiques de type erreur absolue moyenne (MAE), erreur quadratique moyenne (RMSE) et le paramètre Skill (WILLMOTT, 1981 ; WILLMOT et al., 2005).

Le modèle validé est exploité pour simuler des conditions hydrodynamiques caractéristiques de marées de type morte-eau (ME) et vive-eau (VE) moyennes de coefficients respectifs 45 et 95 . Les résultats ont été comparés statistiquement aux prédictions et roses de courants du SHOM issues de l'analyse de mesures.

Mots-clés : Hydrodynamique côtière, Modélisation tridimensionnelle, Maillage triangulaire mixte, Marée, Courants, Région des Pays de la Loire.

\section{Introduction}

Une convention de partenariat, portant sur l'acquisition de la connaissance du milieu marin sur le littoral des Pays de la Loire pour favoriser l'exploitation des EMR, est à l'origine d'un modèle hydrodynamique. Pour certaines formes d'EMR, la connaissance des courants marins sur toute la colonne d'eau revêt un caractère primordial. Le SHOM a ainsi créé un modèle numérique courantologique 3D, afin de représenter correctement la propagation de la marée, et le courant induit, du large à la côte de la région. L’étude 
concerne uniquement la partie maritime, mais pour prendre en compte la dynamique côtière dans son ensemble, l'estuaire de la Loire est intégré jusqu'à la limite de remontée de l'onde de marée, ainsi qu'une partie du littoral morbihannais.

Cet article décrit le modèle hydrodynamique 3D mis en place et l'analyse des résultats du modèle à des mesures in situ récentes et prédictions du SHOM. Il présente aussi la modélisation des marées types ME et VE moyennes, finalité du modèle.

\section{Matériel et méthodes}

\subsection{Description du modèle numérique 3D}

Les résultats présentés sont obtenus à partir du code de calcul TELEMAC-3D, développé par le LNHE d'EDF-R\&D (EDF, 2013). Ce code résout, sur un maillage triangulaire mixte, aux éléments finis, les équations de Navier-Stokes à trois dimensions, en condition de pression hydrostatique (HERVOUET, 2003).

Le maillage horizontal se compose de 12261 nœuds, voir figure 1. La taille des mailles varie entre $7 \mathrm{~km}$ au large, près de la frontière, et 50 à $100 \mathrm{~m}$ à l'approche du littoral des Pays de la Loire et dans l'estuaire de la Loire, zones les plus raffinées. Un système de lignes de contraintes sur lequel le maillage s'appuie permet de bien représenter les gradients de bathymétrie et les directions privilégiées de l'écoulement. Sur la verticale le maillage est dupliqué sur 13 plans horizontaux sigma, répartis suivant des proportions données, rapprochés en surface et au fond et compte près de 160000 nœuds de calcul.

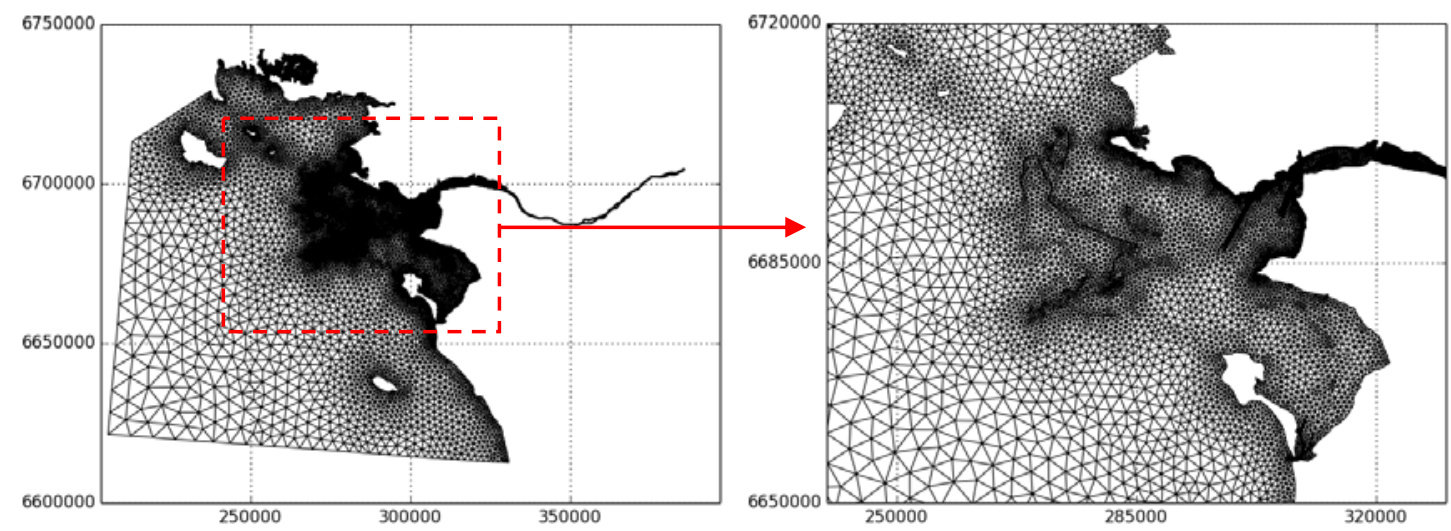

Figure 1. Maillage triangulaire mixte et zoom sur la rade de la Loire.

La bathymétrie est constituée des sondes bathymétriques issues de la Base de Données Bathymétriques du SHOM (BDBS), pour la partie maritime, et du Modèle Numérique de Terrain Unifié (MNTU) dans l'estuaire de la Loire et d'un MNT simplifié du fleuve à l'amont de Nantes (propriété du Groupement d'Intérêt Public Loire Estuaire). 


\section{XIII ${ }^{\text {èmes }}$ Journées Nationales Génie Côtier - Génie Civil \\ Dunkerque, 2-4 juillet 2014}

L’interpolation des données de bathymétrie avec le maillage abouti au MNT, figure 2, qui sert de base aux simulations numériques.

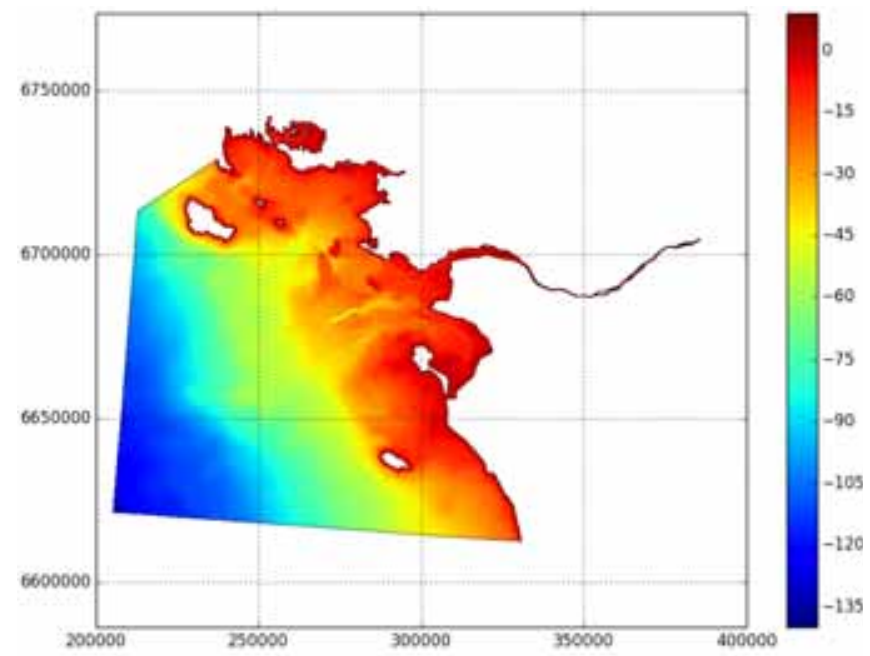

Figure 2. MNT (bathymétrie en mètres par rapport au niveau moyen).

Afin de simuler la dynamique côtière et estuarienne, le modèle est forcé en marée et en débit. Il est forcé, à sa limite maritime par les niveaux d'eau issus du modèle de marée cstFRANCE du SHOM et, à sa limite fluviale par le débit réel de la Loire issu des données de la Banque Hydro.

Plusieurs tests de sensibilité ont été effectués afin d'obtenir un modèle stable et un calage satisfaisant pour les besoins de l'étude. Ils ont porté principalement sur le maillage, le frottement sur le fond variable en espace, les conditions de forçage, le modèle de turbulence, la condition de convergence CFL (Courant Friedrichs Lewy), etc. Le frottement sur le fond est paramétré selon la loi de Chézy. En milieu maritime, le frottement présente des enjeux plus faibles qu'en zone estuarienne notamment du fait de hauteurs d'eau plus importantes. La propagation de la marée est essentiellement contrôlée par des effets topographiques. La modification du coefficient de frottement ne modifie pas de façon manifeste l'élévation de surface dans la partie maritime. Elle est plus significative dans la partie estuarienne et au niveau des zones découvrantes du fait de la faible hauteur d'eau.

\subsection{Données et période de validation}

La validation du modèle est réalisée à la fois en niveau d'eau et en vitesse sur une période réelle. La période retenue est octobre 2011, période d'étiage de la Loire, car présente des données in situ récentes et fiables sur la zone d'étude. Les données de références utilisées pour le calage hydrodynamique sont de deux types : 
a) Mesures in situ (localisées sur la figure 3a) :

- observations du niveau d'eau issues des marégraphes du SHOM mais aussi de partenaires REFMAR tel que le Grand Port Maritime de Nantes Saint-Nazaire,

- mesures de courants observés par des courantomètres de type ADCP (Acoustic Doppler Current Profiler) lors de campagnes en mer réalisées par le SHOM.

b) Prédictions de marée du SHOM, disponibles aux 30 stations (localisées sur la figure 3b) issues de l'analyse harmonique des mesures.

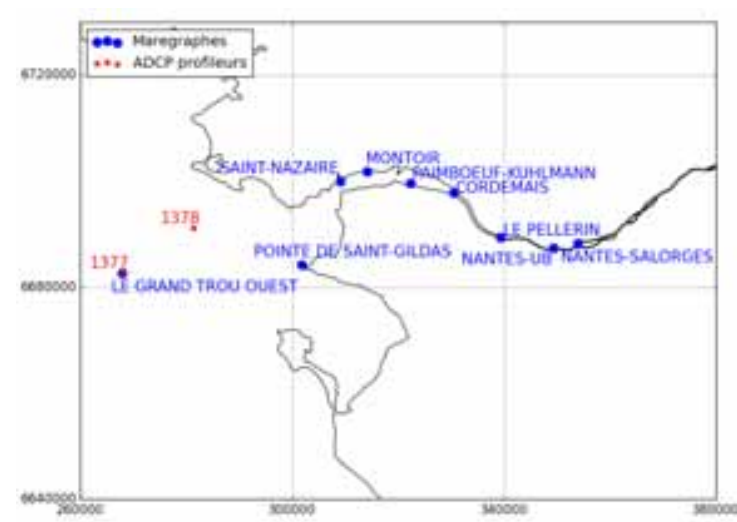

(a)

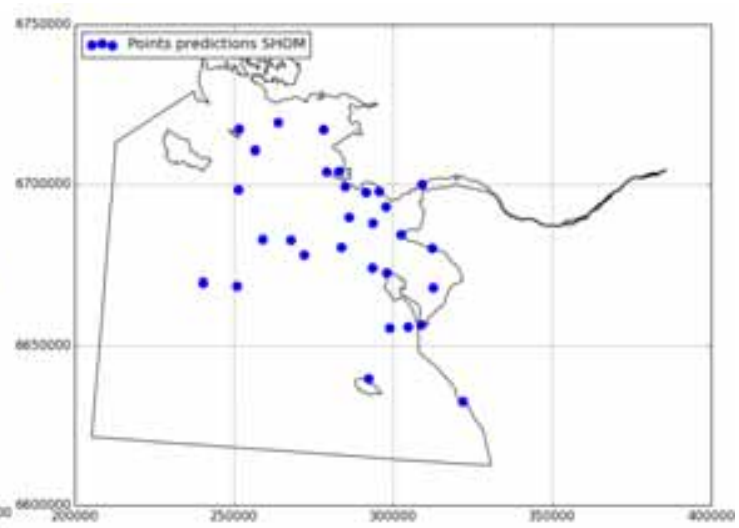

(b)

Figure 3. Localisation des points de validation (a) Mesures in situ (b) Prédictions.

\section{Résultats}

\subsection{Analyse quantitative du modèle}

Le calage hydrodynamique est quantifié par des méthodes statistiques d'erreur absolue moyenne (MAE) et d'erreur moyenne quadratique (RMSE). Leurs valeurs ne peuvent être que positives et, plus elles tendent vers 0 moins l'erreur commise est importante. Un paramètre Skill, équation (1), appelé "index of agreement" (WILLMOTT et al., 2012), et récemment utilisé dans différentes études de dynamique côtière et estuarienne (WARNER et al., 2005 ; TOUBLANC et al., 2013) est calculé. Il s'agit d'un estimateur du degré du résultat du modèle sans erreurs, plus il tend vers 1 moins l'erreur commise est importante.

$$
\text { Skill }=1-\frac{\sum_{i=1}^{n}\left|X_{\text {model }}-X_{\text {obs }}\right|^{2}}{\sum_{i=1}^{n}\left(\left|X_{\text {model }}-\bar{X}_{\text {obs }}\right|+\left|X_{\text {obs }}-\bar{X}_{\text {obs }}\right|^{2}\right.}
$$

Les résultats de l'analyse statistique des niveaux d'eau et des courants sont présentés pour une période de 15 jours, du 02 au 16 octobre 2011. 


\section{XIII ${ }^{\text {èmes }}$ Journées Nationales Génie Côtier - Génie Civil \\ Dunkerque, 2-4 juillet 2014}

\subsection{Niveaux d'eau}

Le modèle reproduit bien l'oscillation verticale du niveau d'eau sur toute l'emprise maritime avec une erreur moyenne RMSE de $0.10 \mathrm{~m}$ et un paramètre Skill de 0.9988, voir tableau 1, entre les 30 points de prédictions du SHOM et le modèle. L'erreur maximale se situe au niveau de la zone découvrante de la baie de Bourgneuf.

Les mesures aux marégraphes sont aussi bien simulées au niveau du Grand Trou Ouest et jusqu'à la partie aval de Cordemais, voir tableau 1 et figure 4. L'évolution de l'intrusion marine est bien reproduite sur le bief qui contribue le plus au stockage d'eau, de Saint-Nazaire à Cordemais.

La partie fluviale n'étant pas la zone d'intérêt de l'étude, une diminution de l'amplitude de marée par rapport aux mesures, basses mers difficilement atteintes contrairement aux pleines mers, en amont de Le Pellerin est acceptable. Les écarts significatifs de RMSE et MAE, au Pellerin et aux stations de Nantes, sont expliqués par la faible résolution du maillage en zone fluviale, la difficulté de reproduire les conditions de frottement en période d'étiage et le MNTU ne correspond pas au lit incisé de la Loire en période d'étiage.

Tableau 1. Comparaisons données de référence et modèle pour le niveau d'eau.

\begin{tabular}{lllll}
\hline Niveau d'eau & Station & MAE $(\boldsymbol{m})$ & $\boldsymbol{R M S E}(\boldsymbol{m})$ & Skill \\
\hline (Obs/modèle) & Le Grand Trou Ouest & 0.06 & 0.08 & 0.9983 \\
& Pointe de Saint-Gildas & 0.14 & 0.17 & 0.9980 \\
& Saint-Nazaire & 0.13 & 0.16 & 0.9973 \\
& Montoir & 0.10 & 0.13 & 0.9978 \\
& Paimboeuf-Kuhlmann & 0.10 & 0.13 & 0.9977 \\
& Cordemais & 0.13 & 0.16 & 0.9965 \\
& Le Pellerin & 0.22 & 0.27 & 0.9902 \\
& Nantes-Usine Brulée & 0.45 & 0.54 & 0.9598 \\
& Nantes-Salorges & 0.53 & 0.62 & 0.9454 \\
\hline (Préd/modèle) & Moyenne sur 30 stations & 0.08 & 0.10 & 0.9988 \\
\hline
\end{tabular}

Les écarts révélés par le paramètre Skill sont ici toujours faibles. Par exemple sur la station Nantes-Salorges le paramètre Skill est de 0.945, soit proche de 1, ce qui montre que les écarts observations/modèle sont faibles. La RMSE quant à elle présente un écart significatif de $0.62 \mathrm{~m}$, d'où l'utilité de quantifier l'erreur par diverses approches statistiques. 


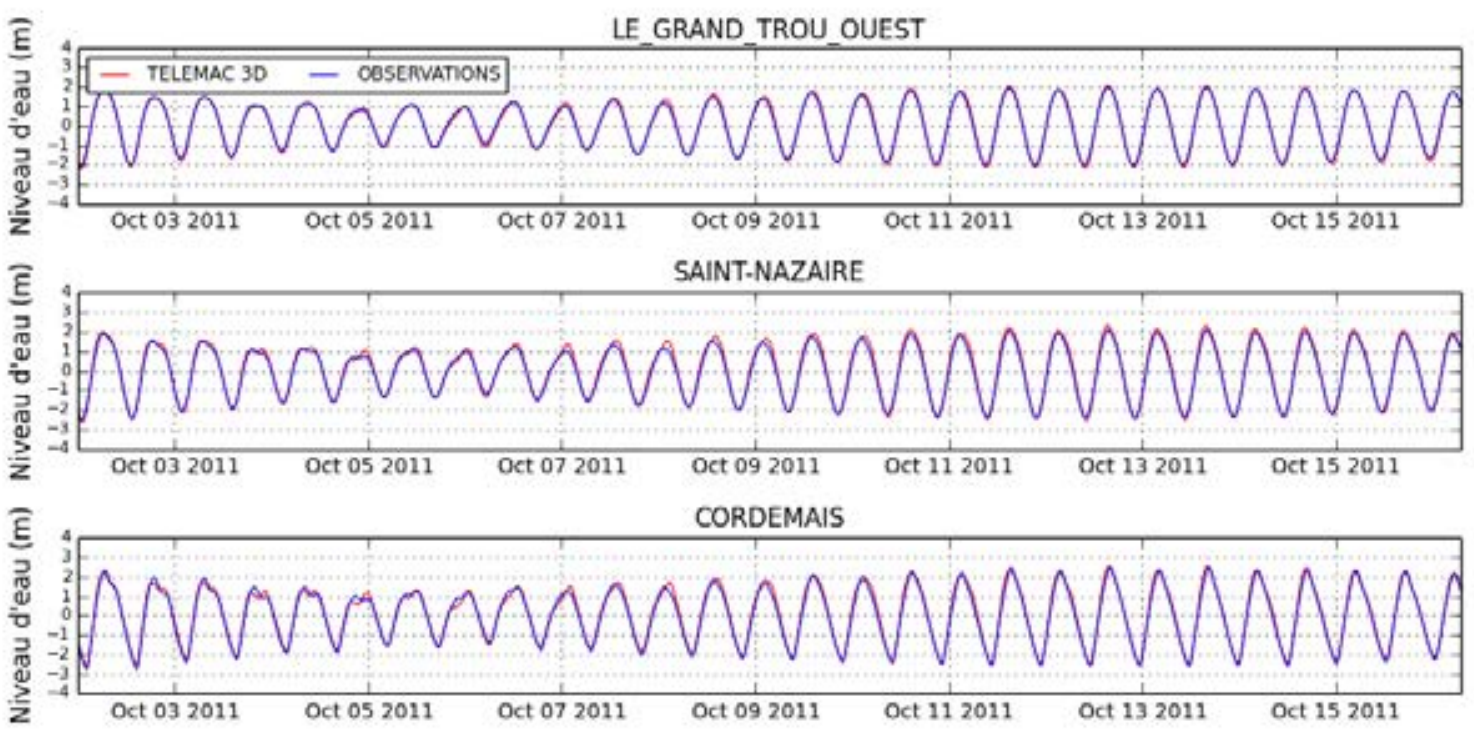

Figure 4. Comparaisons des niveaux d'eau entre les observations marégraphiques (bleu) et le modèle TELEMAC-3D (rouge).

\subsection{Courants}

Le modèle reproduit correctement la composante de vitesse zonale $U$ sur toute la colonne d'eau, aux deux points de mesures ADCP, voir tableau 2. $U$ correspond à la composante principale du courant de marée sur le littoral Loire-Atlantique, direction du courant principalement orienté d'Ouest en Est. Lors de la période de ME, aux alentours du 7 octobre, le modèle au point de station 1377 sous-estime en valeur absolue la composante de vitesse méridionale $V$, voir figure 5 . Le phénomène est accentué pour la station 1378 et est présent aussi sur son cycle de VE mais de façon réduite. Cette sousestimation au niveau de la composante $V$, en ME, explique les faibles valeurs obtenues pour le paramètre Skill contrairement à la composante $U$. Les écarts de vitesse constatés à la station 1378, située à l'Ouest du Plateau de la Banche, peuvent être dus à une trop faible discrétisation verticale dans une zone de gradients bathymétriques élevés.

Le paramètre Skill permet ici de rendre compte d'erreurs non révélées par la MAE et la RMSE. Les méthodes de quantification de l'erreur ne semblent pas toujours cohérentes entre elles, d'où l’intérêt de la quantifier par différentes approches statistiques.

Tableau 2. Comparaisons mesures et modèle pour les composantes de vitesse $U$ et $V$.

\begin{tabular}{lllll}
\hline Vitesse & $\begin{array}{l}\text { Station } \\
\text { [immersion en m] }\end{array}$ & MAE $(\mathbf{m} / \mathbf{s})$ & $\boldsymbol{R M S E}(\mathbf{m} / \mathbf{s})$ & Skill \\
\hline Composantes & $1377[9,5 \mathrm{~m}]$ & $0.06 ; 0.09$ & $0.08 ; 0.11$ & $0.9548 ; 0.8388$ \\
de & $1377[17 \mathrm{~m}]$ & $0.05 ; 0.08$ & $0.06 ; 0.10$ & $0.9720 ; 0.8458$ \\
vitesse & $1377[24,5 \mathrm{~m}]$ & $0.04 ; 0.07$ & $0.05 ; 0.09$ & $0.9761 ; 0.8091$ \\
$(\mathrm{U} ; \mathrm{V})$ & $1378[9 \mathrm{~m}]$ & $0.04 ; 0.11$ & $0.05 ; 0.09$ & $0.9681 ; 0.5605$ \\
& $1378[19 \mathrm{~m}]$ & $0.05 ; 0.07$ & $0.06 ; 0.08$ & $0.9366 ; 0.6320$ \\
\hline
\end{tabular}



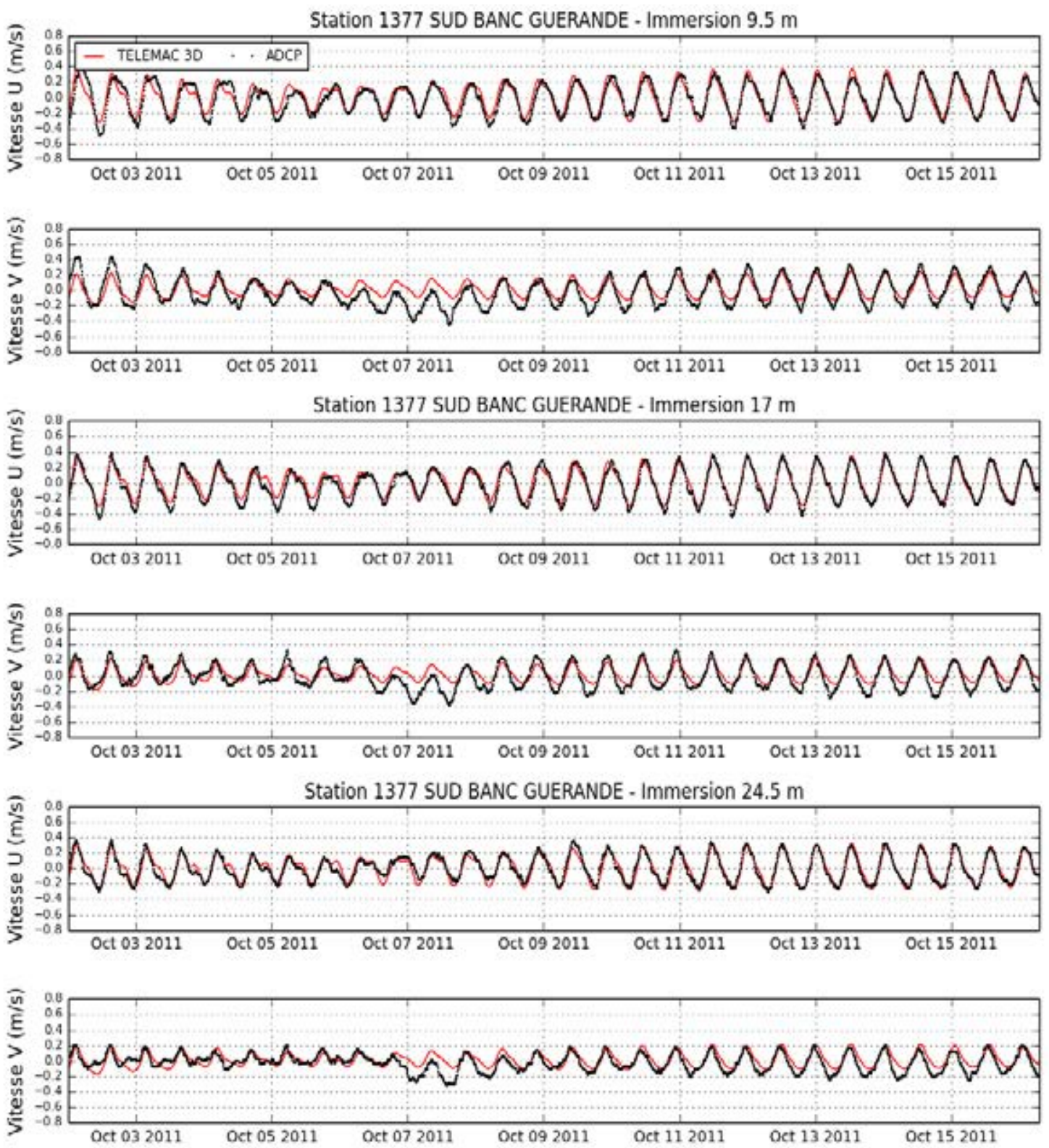

Figure 5. Comparaisons à plusieurs immersions des composantes de vitesse $U$ et $V$ entre les mesures ADCP (noir) et le modèle TELEMAC-3D (rouge) au point 1377.

\section{Exploitation du modèle sur des marées types}

Le modèle ainsi validé est disposé pour simuler des marées types ME et VE moyennes de coefficients respectifs 45 et 95 (NICOLLE \& PINEAU, 2009) sous des conditions de débit moyen de $850 \mathrm{~m}^{3} / \mathrm{s}$. Le signal de marée au forçage est toujours issu du modèle de marée cstFRANCE. Avant d'être exploité les résultats du modèle sont comparés aux données statistiques du SHOM, prédictions du niveau d'eau et roses de courant, issues de l'analyse des mesures (données marégraphiques, courantomètres ponctuels et profileurs) (SIMON, 2007). 
Le modèle reproduit bien les amplitudes de marées types sur toute l'emprise maritime. L'erreur moyenne RMSE, pour les niveaux d'eau aux 30 points localisés figure 3b, est de $0.03 \mathrm{~m}$ en ME et $0.08 \mathrm{~m}$ en VE.

Les courants modélisés sont comparés aux roses de courants du SHOM. Le résultat de comparaison concernant les courantomètres ponctuels sur une marée type VE est présenté figure 6.
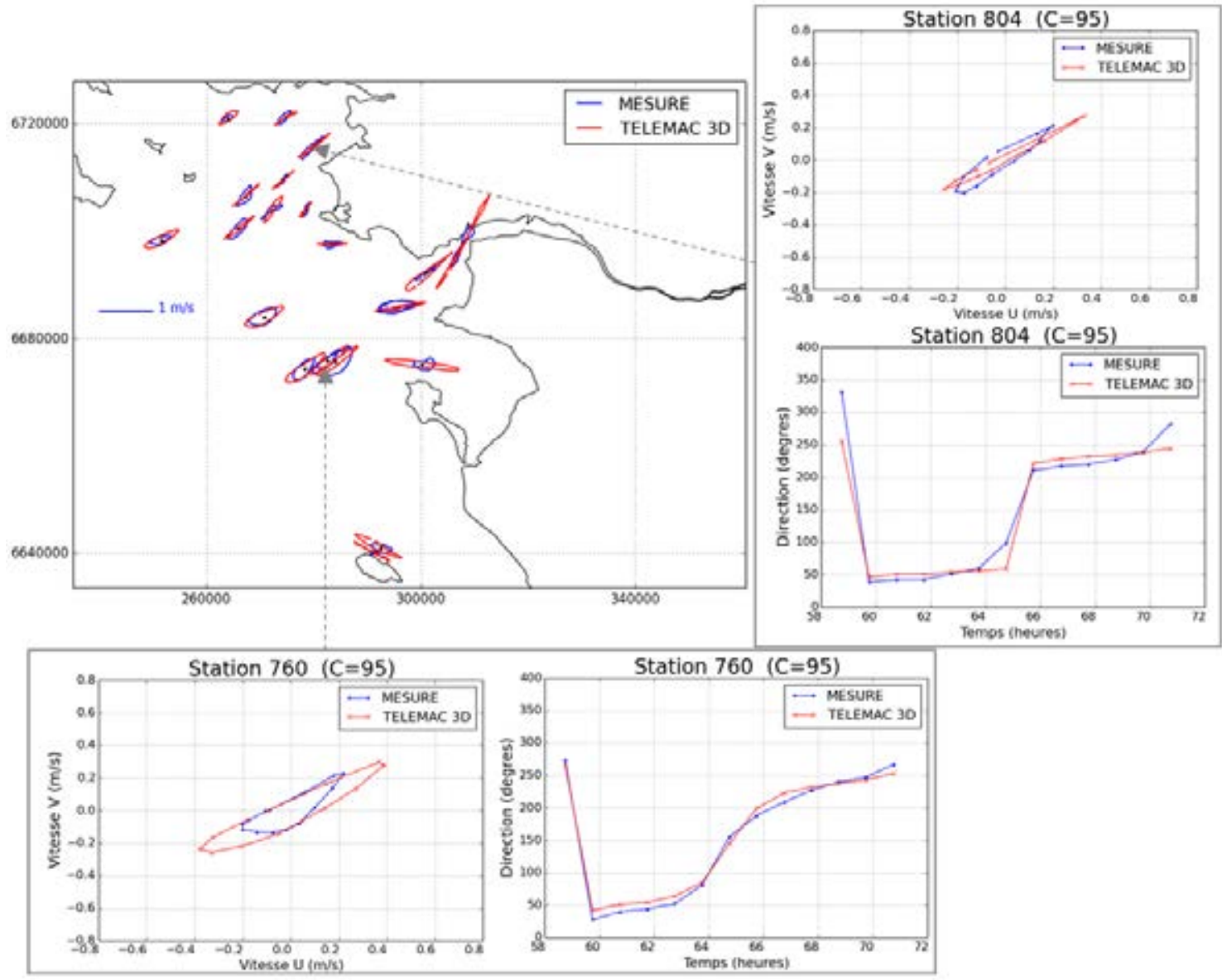

Figure 6. Comparaisons des roses de courant mesurées et modélisées pour une VE.

La dynamique côtière engendrée par les courants de marée est bien représentée par le modèle. Les directions sont correctement reproduites et les heures de renverse correspondent. Les intensités de vitesse des courants sont dans l'ensemble satisfaisantes. Ce sont les courants moyens modélisés sur la colonne qui sont comparés aux roses de courants statistiques issues de l'analyse des mesures de courantomètres ponctuels, ce qui peut expliquer certains écarts en intensité constatés. 


\section{XIII ${ }^{\text {èmes }}$ Journées Nationales Génie Côtier - Génie Civil \\ Dunkerque, 2-4 juillet 2014}

\section{Conclusions}

Le modèle hydrodynamique tridimensionnel de la région des Pays de la Loire est validé en niveau d'eau et en vitesse de courant sur une période réelle récente. L'analyse statistique permet de quantifier les erreurs du modèle par rapport aux mesures in situ et prédictions. Le modèle reproduit correctement la marée sur la partie maritime et littoral de la région et ce jusqu'au niveau de Cordemais dans l'estuaire de la Loire. Les composantes de vitesse sont relativement bien représentées sur toute la colonne d'eau.

Le modèle est exploité pour modéliser des marées types ME et VE moyennes de coefficients respectifs 45 et 95 . Les résultats de simulation sont eux aussi comparés aux données statistiques du SHOM. In fine le modèle permet de caractériser l'évolution des courants de marée sur la colonne d'eau pour l'ensemble du littoral de la région et pas seulement en quelques points isolés. L'application la plus pertinente d'un tel modèle est l'évaluation du potentiel hydrolien d'une région. La connaissance des courants de marée constitue également un apport essentiel pour les autres formes d'EMR mais aussi diverses activités maritimes.

\section{Références bibliographiques}

EDF (2013). Système de modélisation TELEMAC. Logiciel TELEMAC-3D Version 6.2. Manuel de l'utilisateur.

HERVOUET J.M. (2003). Hydrodynamique des écoulements à surface libre. Modélisation numérique avec la méthode des éléments finis. Presses de l'école nationale des Ponts et Chaussées, Paris, 311 p.

NICOLLE A., PINEAU L. (2009). Modélisation numérique hydrodynamique côtière. Guide Technique SHOM n ${ }^{\circ}$ 2007-050.

SIMON B. (2007). La marée océanique côtière. Coédition de l’Institut océanographique et du SHOM, 433 p.

TOUBLANC F., BRENON I., COULOMBIER T., LE MOINE O. (2013). Salinity and suspended dynamics in response to forcing changes in a small macrotidal estuary (Charente, France). Coastal Dynamics, pp 1707-1718.

WARNER J.C., GEYER W.R., LERCZAK J.A. (2005). Numerical modeling of an estuary: A comprehensive skill assessment. Journal of Geophysical research. Vol. 110, C05001 http://dx.doi.org/10.1029/2004JC002691

WILLMOTT C.J. (1981). On the validation of models. Physical Oceanography, Vol. 2(2), pp 184-194.

WILLMOTT C.J., MATSUURA K. (2005). Advantages of the mean absolute error (MAE) over the root mean square error (RMSE) in assessing average model performance. Climate Research, Vol. 30, pp 79-82. http://dx.doi.org/10.3354/cr030079

WILLMOTT C.J., ROBENSON S., MATSUURA K. (2012). Short communication; A refined index of model performance. International Journal of climatology, Vol. 32, pp 2088-2094. http://dx.doi.org/10.1002/joc.2419 
Thème 1 - Hydrodynamique côtière 